\title{
The effect of diluent composition on homogeneous charge compression ignition auto-ignition
}

and combustion duration

Janardhan Kodavasal $^{\text {* }}$, George A. Lavoie ${ }^{\mathrm{a}}$, Dennis N. Assanis ${ }^{\mathrm{b}}$, and Jason B. Martz

a. Walter E Lay Automotive Laboratory, University of Michigan, Ann Arbor, MI, USA

b. Stony Brook University, Stony Brook, NY, USA

*corresponding author (currently with Argonne National Laboratory):

Address-9700 S. Cass Ave., Bldg. 362, Lemont, IL - 60439, USA

Email-jkodavasal@anl.gov

Telephone-(734)546-8210

Fax-(630)252-3443

Submitted to the "IC ENGINE AND GAS TURBINE COMBUSTION" colloquium

Total length of paper using Method 1: 6193.89 words

Breakup of lengths of sections in words:

Main text: 3961.40

References: 437.00

Fig. 1: 117.94

Fig. 2: 142.08

Fig. 3: 143.08

Fig. 4: 263.17

Fig. 5: 160.08

Fig. 6: 151.08

Fig. 7: 152.08

Fig. 8: 155.08

Fig. 9: 146.08

Table 1: 121.60

Table 2: 243.20

All figures may be printed in grayscale 


\section{The effect of diluent composition on homogeneous charge compression ignition auto-ignition and combustion duration}

In this work, the effect of diluent composition is studied on the ignition timing and combustion duration of homogeneous charge compression ignition (HCCI) combustion. Full-cycle 3D computational fluid dynamics (CFD) simulations were performed using two different dilution schemes, one which employs air dilution and the other which employs external exhaust gas recirculation (eEGR) dilution. Both cases used a fixed breathing strategy, and had the same fueling rate and engine speed typical of automotive applications. A premixed fuel-air mixture was used in the intake charge to avoid the fuel stratification effects associated with direct injection, while the valve events were selected to minimize the internal residual and its associated thermal and compositional stratification. With ignition timing held constant near top dead center (TDC) for both methods, there is a $10 \%$ increase in the combustion duration going from air to eEGR dilution. While the thermal stratification prior to ignition is similar for both methods, the eEGR-dilute case has an overall higher temperature throughout the charge. A reactivity stratification analysis, based on the distribution of ignition delays within the charge prior to ignition, showed nearly identical initial reactivity for the two methods, with the higher temperatures of the eEGR case compensating for this method's lower oxygen content. A quasidimensional multi-zone model was subsequently used to decouple the chemical kinetic and thermodynamic effects of eEGR on the combustion process. This analysis showed that the primary factor contributing to the longer combustion duration for the eEGR-dilute case is the lower ratio of specific heats $(\gamma)$ in the unburned charge post-ignition which is thought to lower the rate of compression-induced heating of the unburned charge by the ignited and burning regions, leading to slower sequential auto-ignition.

Keywords: IC Engines; HCCI; EGR; reactivity stratification; CFD.

\section{Introduction}

Homogeneous charge compression ignition (HCCI) is a novel combustion mode that combines many desirable features of the spark-ignited and diesel combustion modes [1], [2]. One major challenge associated with HCCI is the near volumetric combustion event which results in high rates of energy release relative to modes utilizing premixed or diffusion flames. Dilution of the charge by introducing excess air (compared to stoichiometric) or by introducing exhaust gases into the cylinder, serves to reduce rates of energy release as well as peak combustion temperatures.

One common method for charge dilution in HCCI is through the use of external exhaust gas recirculation (eEGR), where the products of combustion from the previous cycle are intentionally recirculated typically by extracting them from the exhaust runner, passing them through a cooler to control their temperature, and then finally mixing them with the intake air at the intake runner. The differing thermodynamic and chemical properties of EGR and air affect combustion characteristics such as phasing, rate and emissions. Early HCCI studies which investigated the effect of diluent composition, [3] - [5] showed that with all other parameters being held the same, increasing the 
fraction of eEGR within the charge retarded ignition timing while increasing combustion duration. The later phasings were due in part to the lower end-of-compression temperatures associated with the increased mixture heat capacity, which reduced the mixture specific heat ratio $\left(C_{p} / C_{v}, \gamma\right)$, resulting from the larger fraction of tri-atomic $\mathrm{H}_{2} \mathrm{O}$ and $\mathrm{CO}_{2}$ molecules within the EGR dilute mixture.

In their 2009 work, Dec et al. [6] showed that addition of eEGR resulted in a small reduction in the peak heat release rate (HRR) when combustion phasing (represented by the crank angle of 50\% burn, CA50) was matched. Olsson et al. [4] reached similar conclusions, finding that the effect of replacing air with eEGR was small in terms of HRR when combustion phasing was matched.

Despite the above work, the mechanism behind the increased combustion duration at a given combustion phasing with eEGR remains unclear. While some homogeneous reactor studies have been performed to better understand the chemical and thermal factors associated with the use of EGR [7], these studies are only applicable to the ignition event. Rapid compression and shock tube studies similarly concentrate on the ignition event while the combustion itself is much faster than typically observed in engines. This difference is thought to be due to the thermal and compositional stratification in engines. It is therefore of interest to use more detailed models to better understand the fundamental impact of dilution method on combustion characteristics such as combustion duration for a given ignition timing.

In the current work, full-cycle reacting CFD simulations were performed with gasoline kinetics to investigate the impact of air as well as eEGR dilution on HCCI combustion. The reason why full-cycle simulations are needed is to identify potential stratification effects arising from trapped residuals which cannot be captured by closed-cycle simulations such as those used in previous EGR studies [8]. Fueling and ignition timing were maintained constant to remove the impact of these variables from the combustion process. Reaction space analysis, based on the distribution of ignition delays within the charge prior to ignition, was performed on the CFD results to determine the effects of dilution method on the pre-ignition reactivity stratification. Finally, a quasi-dimensional (Quasi-D) multi-zone model was used to decouple the chemical and thermodynamic effects of air vs. eEGR dilution on combustion duration. Detailed description and evaluation of both the CFD model as well as the Quasi-D multi-zone model used in the current work are given in [9]. 


\section{CFD predictions for the dilution method study}

To compare air vs. eEGR dilution, full-cycle reacting CFD simulations (starting from $80^{\circ} \mathrm{CA}$ aTDC of the previous cycle and simulating the exhaust and intake strokes) were performed at 2000 revolutions per minute (RPM), with the engine mesh shown in Figure 1. This mesh is based on a fully flexible valve actuation (FFVA) engine on which several HCCI and spark assisted compression ignition (SACI) experiments have been performed [10], [11]. The engine specifications are given in Table 1. The standard k- $\varepsilon$ turbulence model is used with law of the wall. Constant and uniform temperatures were prescribed for the head $(450 \mathrm{~K})$, liner $(450 \mathrm{~K})$ and piston $(480 \mathrm{~K})$. There is no crevice treatment in this study. Multiple cycles were not run to reach convergence. Positive valve overlap (PVO) with negligible overlap was used in this study to minimize the possible stratification effects caused by the retention of internal residuals from the previous cycle [12]. The internal residuals trapped within the clearance volume contribute to approximately $7 \%$ of the total charge mass in this study for both the air and eEGR cases. To avoid any possible stratification effects from direct injection, both cases use a fully-premixed fuel-air or fuel-air-eEGR composition imposed at the intake runner, which is representative of port fuel injection (PFI). The fuel is represented by a four-component gasoline surrogate [13] comprised of isooctane, n-heptane, toluene and 2-pentene. The chemical kinetic mechanism used [13] consists of 312 species and chemistry is solved using the multi-zone methodology outlined in [14] and [15] where zones were formed based on $\Delta T_{\text {zone }}<5 \mathrm{~K}$ and $\Delta \varphi_{\text {zone }}<0.03$ similar to the resolution used in [16]. The equivalence ratio $\varphi$ in this case is the "progress equivalence ratio" defined in [14]. Throughout this work, the composition of eEGR is taken to be products of complete combustion. Nitric oxide (NO) and nitrogen dioxide $\left(\mathrm{NO}_{2}\right)$ remaining from the previous cycle or in eEGR are known to have a sensitizing effect on ignition, and potentially could have an influence on these calculations. Experiments in an HCCI engine have shown that this effect is small, resulting in advances in ignition phasing of less than 2 crank angle degrees (CAD) for up to 500 parts per million (ppm) added intake nitric oxide [17]. Exhaust NO emission levels with HCCI are generally below 100 ppm, so even with 50\% EGR, the intake NO is less than $50 \mathrm{ppm}$. In the eEGR case studied in this work, the concentration of $\mathrm{NO}$ at $40^{\circ} \mathrm{CA}$ aTDC (until which point the simulations were run) is under $3 \mathrm{ppm}$. Further, the kinetic mechanism is complex, involving interactions of nitric oxides with fuel molecules, and is only partially understood at this time [18]. For these reasons the effect is not considered here. Additionally, trace species such 
as $\mathrm{OH}$ have also not been considered in the eEGR composition. Sjöberg et al. [5] compared the effect of using simulated eEGR (consisting of products of complete combustion) to that of using real eEGR (potentially containing trace species) under gasoline HCCI operation and found that the main effect of eEGR is to retard ignition timing due to the cooling effect. Real eEGR tends to advance ignition timing with respect to simulated eEGR, but this was by less than 2 CAD for the conditions studied.

The intake composition in each case was set to achieve $9.3 \mathrm{mg}$ of fuel trapped within the cylinder at intake valve closing (IVC), so that both cases have the same fuel-energy content available for combustion. For the air-dilute case, the resulting in-cylinder fuel to air equivalence ratio $(\Phi)$ was 0.34. For the eEGR case, a fraction of the intake air is replaced by products of complete combustion (which also include excess air). At IVC, the in-cylinder $\Phi$ is 0.61 , while the overall residual gas fraction (RGF) is $43 \%$, a combination of $7 \%$ internal residual (similar to the air-dilute case) and $36 \%$ externally imposed EGR. The overall dilution level for both cases is similar, as the fuel to charge equivalence ratio $\left(\Phi^{\prime}\right)$ [19], which accounts for the impact of both air and EGR dilution on the charge energy was maintained at $\Phi^{\prime} \sim 0.3$. This equivalence ratio is evaluated as follows:

$\Phi^{\prime}=\frac{\left.\left\{\text { mass }_{\text {fuel }} / \text { mass }_{\text {air }}+\text { mass }_{\text {residual }+e E G R}\right)\right\}}{\left\{\text { mass }_{\text {fuel }} / \text { mass }_{\text {air }}\right\}_{\text {stoic. }}} \cong \Phi(1-\mathrm{RGF})$

where $\Phi$ refers to the fuel-air equivalence ratio.

The air-dilute case has a mole-percentage of oxygen $\left(\chi_{O 2}\right)$ of approximately $20 \%$ while the eEGR-dilute case has $\chi_{O 2} \sim 15 \%$. This difference causes the overall fuel-oxygen equivalence ratios $\left(\phi_{F O}\right)$ to vary for the two cases; $\phi_{F O}=0.33$ for the air-dilute case and $\phi_{F O}=0.44$ for the eEGRdilute case. Due to the low levels of hot internal residual, both cases required significant heating of the intake charge in order to achieve ignition timing defined by CA10 at $\sim 1^{\circ} \mathrm{CA}$ bTDC. In addition, the intake temperature of the eEGR case $\left(T_{\text {in }}=241^{\circ} \mathrm{C}\right)$ was higher compared to the air-dilute case $\left(T_{\text {in }}=213^{\circ} \mathrm{C}\right)$. To compensate for this and maintain similar compression pressures and in-cylinder mass, the intake pressure for the eEGR case was raised by 0.0375 bar (over the nominal value of 1 bar) compared to the air-dilute case.

Figure 2 compares the in-cylinder pressure traces for the two cases. The eEGR-dilute case has a slightly lower pressure rise rate and a lower peak pressure, corresponding to the slower burn 
profiles for the eEGR-dilute case shown in Figure 3. The combustion duration (CA10-90) for the airdilute case is $5.0^{\circ} \mathrm{CA}$, while for the eEGR-dilute case is $10 \%$ longer at $5.5^{\circ} \mathrm{CA}$. These observations are in agreement with those from Dec et al. [6] who found a small reduction in energy release rates when replacing air dilution with eEGR dilution.

\section{Analysis of the pre-ignition reaction space from CFD simulation (at $10^{\circ} \mathrm{CA}$ bTDC)}

To understand the reasons for the differences in combustion duration, the ignition delay will be used to assess how the charge reactivity is affected by variations within the local charge state. Consider the He et al. [20] correlation for the ignition delay of isooctane under typical HCCI conditions:

$\tau_{\text {ign }}=1.3 \times 10^{-4} \cdot P^{-1.05} \cdot \phi_{F O}^{-0.77} \cdot \chi_{O_{2}}^{-1.41} \cdot e^{\frac{33700}{R T}}$,

where $\tau_{i g n}$ represents the ignition delay in milliseconds, $P$ represents the pressure in atmospheres, $\phi_{F O}$ represents the fuel-oxygen equivalence ratio, $\chi_{O 2}$ represents the oxygen mole percentage, $R$ is the universal gas constant in cal $/ \mathrm{K} / \mathrm{mol}$ and $T$ is the temperature in Kelvin. While more complex ignition delay expressions are available which include negative temperature coefficient (NTC) behavior [21], this simpler expression in pure Arrhenius form, is preferred for the clarity it offers regarding the relative importance of the key variables. Ignition delays calculated with the full kinetic mechanism at $-10^{\circ} \mathrm{CA}$ aTDC, closely duplicate the He et al., expression for temperatures above $1000 \mathrm{~K}$, in a range which includes over $90 \%$ of the mass. Thus the simpler expression will be used in what follows.

Figure 4 shows the reaction space for both cases at $10^{\circ} \mathrm{CA}$ bTDC when $\sim 1 \%$ of the charge is burned (CA1). The $x$-axis of the reaction space is spanned by $T$ while the $y$-axis is spanned by $\phi_{F O}$; $\chi_{\mathrm{O} 2}$ is represented by a grey-scale. It is apparent that both the air-dilute and eEGR-dilute cases have negligible compositional stratification. The charge thermal stratification is computed based on two standard deviations $(2 \sigma)$ in charge temperature. The standard deviation is computed on a moleweighted basis as follows: 
$\sigma T=\sqrt{\frac{\sum_{i=1}^{N} n_{\mathrm{i}}\left(T_{i}-\bar{T}\right)^{2}}{\left(\frac{N-1}{N}\right) \sum_{i=1}^{N} n_{\mathrm{i}}}}$

where " $i$ " is the subscript denoting a CFD cell, " $n$ " represents number of moles, " $N$ " represents the total number of CFD cells, and $\bar{T}$ is the mole-weighted average temperature within the CFD domain. Thermal stratification, expressed as $2 \sigma T$, is $74 \mathrm{~K}$ for the air-dilute case and $78 \mathrm{~K}$ for the eEGR-dilute case. The $2 \sigma$ stratification in $\phi_{F O}$ is 0.002 for the air-dilute case and 0.004 for the eEGR-dilute case. The $2 \sigma$ stratification in $\chi_{O 2}$ is $0.2 \%$ for the air-dilute case, and $0.12 \%$ for the eEGR-dilute case. Eq. (2) is used with the mean nominal pre-ignition conditions at $10^{\circ} \mathrm{CA}$ bTDC for the air case $\left(P=24\right.$ bar, $\phi_{F O}=0.33, \chi_{O 2}=19.9 \%$ and $\left.T=1045 \mathrm{~K}\right)$ and for the eEGR case $(P=24$ bar, $\phi_{F O}=0.44, \chi_{O 2}=15.1 \%$ and $T=1058 \mathrm{~K}$ ). Corresponding to the $2 \sigma$ stratifications in the three reaction space dimensions for the air-dilute case, an increase in $\phi_{F O}$ of 0.002 produces a $0.46 \%$ reduction in ignition delay, an increase in $\chi_{O 2}$ of $0.2 \%$ produces a $1.40 \%$ reduction in ignition delay, and an increase in $T$ of $74 \mathrm{~K}$ produces a $65.83 \%$ reduction in ignition delay, as computed using Eq. (2). Corresponding to the $2 \sigma$ stratifications in the three reaction space dimensions for the eEGRdilute case, an increase in $\phi_{F O}$ of 0.004 produces a $0.69 \%$ reduction in ignition delay, an increase in $\chi_{O 2}$ of $0.12 \%$ produces a $1.11 \%$ reduction in ignition delay, and an increase in $T$ of $78 \mathrm{~K}$ produces a $66.76 \%$ reduction in ignition delay. Thus, in terms of affecting ignition delays, the stratification in $\phi_{F O}$ for both air- and eEGR-dilute cases is roughly two orders of magnitude smaller than the stratification in $T$, and the stratification in $\chi_{O 2}$ is at least 47 times smaller than the stratification in $T$. Clearly, the level of stratification in the composition dimensions of $\phi_{F O}$ and $\chi_{O 2}$ is insufficient in both the air-dilute and eEGR-dilute cases to have a significant impact on the overall reactivity stratification (the stratification in ignition delays within the charge). Reactivity stratification is therefore primarily dominated by thermal stratification in both cases. It must be noted that these predictions of thermal stratification are higher than those computed by Sandia researchers [22] which were on the order of $27 \mathrm{~K}$ in terms of $2 \sigma T$ near TDC. This is possibly because the Sandia researchers compute the thermal stratification based on a "pixel" or "area" weighted standard deviation over a 2D plane, whereas the thermal stratification our CFD study is computed based on the entire 3-D domain as a mole-weighted $2 \sigma T$. It is also likely that the Sandia engine, which is based on a diesel 
engine with 14 compression ratio, has different heat loss characteristics compared to the pent-roof gasoline engine (12.5 compression ratio) used in this study. It is our experience from previous CFD studies [23] involving both the Sandia engine and the FFVA engine used in this work that the Sandia engine has shorter burn durations, possibly due to the lower thermal stratification in that engine.

Considering the similar thermal stratification observed with air or eEGR dilution, and the minimal importance of compositional stratification predicted for the conditions within the current work, the focus is now shifted to other factors that could affect burn rate. It is important to note that the mean temperature for the eEGR case at pre-ignition in Figure 4 is $14 \mathrm{~K}$ higher than that with air dilution, indicating that the unburned temperature needed to match ignition timing for the eEGR case is higher than that for the air-dilute case. This difference is also apparent in the thermal stratification within the CFD simulations for each dilution method in Figure 5 at $10^{\circ} \mathrm{CA}$ bTDC, which shows the cumulative mass fraction above a given temperature. Note that the temperature axis in this figure $(x$ axis) is in the reverse direction going from hot to cold. The high temperature leftmost edge of the temperature distribution in Figure 5 is shifted to lower temperatures for the air-dilute case. Furthermore, the overall temperature distribution is also shifted to the right (colder) for the air-dilute case compared to the eEGR-dilute case. Despite this shifting in unburned gas temperature, the overall thermal stratification for both cases is almost identical. Additionally, the ignition timing for both cases in Figure 3 is well matched. To better understand this behavior, we compare the "reactivity stratification" based on the ignition delay computed in every CFD cell using eq. (2). In this work, locally computed ignition delays throughout the charge are used to represent reactivity distribution. For consistency with the $\mathrm{He}$ et al. expression, the ignition delay calculations are performed with data from non-reacting CFD simulations, which share the same initial conditions as the reacting simulations. The cumulative reactivity distribution calculated with the ignition delay in every CFD cell is shown in Figure 6. The air-dilute and eEGR-dilute cases have well-matched reactivity distribution throughout the charge, including the high temperature leading edge which controls ignition timing. This results from the higher temperature of the eEGR case compensating for the deficiency in charge oxygen, in effect causing the ignition delay to be matched for the hottest $10 \%$ of the charge mass as well as the rest of the charge. However, the combustion duration for the eEGR case is about $10 \%$ longer (Figure 3) than that of the air-dilute case, in-spite of having similar pre-ignition reactivity distribution. 


\section{Decoupling oxygen effects and thermodynamic effects on combustion duration}

Considering the fact that the reactivity distribution is well matched, it can be hypothesized that the differences in combustion duration may result from thermodynamic property variations associated with the dilution methods. Figure 7 shows the distribution of the ratio of specific heats $(\gamma)$ for the whole CFD domain for both air and eEGR dilution. Both cases have a higher $\gamma$ in regions with longer ignition delays, which is expected given that $\gamma$ decreases with temperature for a given composition. More interesting to note however, is that the $\gamma$ for the eEGR-dilute case is lower than that for the air-dilute case over the whole ignition delay space. Considering that $\gamma$ may influence the polytropic compression work transfer from the earlier to later burning regions, it is feasible that the lower $\gamma$ of the eEGR case leads to a slower rate of increase in both the end-gas temperature and reactivity of the later burning regions of the eEGR-dilute charge.

To test this hypothesis, a Quasi-D multi-zone model developed by Kodavasal et al. [23] is adapted to isolate the effect of $\gamma$. This model employs a series of discrete zones which interact with each other by pressure work only, and once initialized the zones are adiabatic. To initialize the Quasi-D model, a non-reacting CFD simulation is run starting at $80^{\circ} \mathrm{CA}$ aTDC of the previous cycle; through the exhaust and intake processes, up to $10^{\circ} \mathrm{CA}$ bTDC (pre-ignition) of the cycle being studied. The pre-ignition CFD domain is then binned in terms of $T, \chi_{O 2}$ and $\phi_{F O}$ so that the $\Delta T_{\text {bin }}<5$ $\mathrm{K}, \Delta \chi_{O 2 b i n}<2 \%$ and $\Delta \phi_{F O}<0.05$. This results in the creation of 88 bins for the air-dilute case and 55 bins for the eEGR-dilute case. These resolutions are chosen to minimize the error between ignition delay distributions from the $\mathrm{He}$ et al. correlation computed using the average CFD bin properties and those computed within every CFD cell at $10^{\circ} \mathrm{CA}$ bTDC. Every bin from the nonreacting CFD simulation is then used to create a corresponding zone for the Quasi-D model, and information such as species masses and temperature from each CFD bin is mapped onto the corresponding Quasi-D zone. Thus, the non-reacting CFD calculation is used only to initialize the Quasi-D model which then solves kinetics. The Quasi-D model is run with the same gasoline mechanism used in the reacting CFD simulations. A consistent positive shift on the order of $15 \mathrm{~K}$ to the initial temperature profiles for both the air-dilute and eEGR-dilute cases was needed to attain the same ignition timing as the reacting CFD case possibly because the Quasi-D approach (which is initialized from a non-reacting CFD simulation) does not account for intermediates and fuel breakdown which would occur in the reacting CFD simulation by $10^{\circ} \mathrm{CA}$ bTDC. 
In order to decouple the $\gamma$ effect, a third diagnostic case is introduced (called $\mathrm{N}_{2}$-dilute) that is identical to the eEGR case, except that when initializing the Quasi-D model at $10^{\circ} \mathrm{CA}$ bTDC, a portion of burned gas $\left(\mathrm{CO}_{2}\right.$ and $\left.\mathrm{H}_{2} \mathrm{O}\right)$ mass is replaced in every zone of the Quasi-D model with an equal mass of $\mathrm{N}_{2}$, such that the overall mass in the zone is preserved, the mass of oxygen and fuel within the zone are also preserved, and the ratio of the mass of $\mathrm{CO}_{2}$ to the mass of $\mathrm{H}_{2} \mathrm{O}$ within the zone remains constant. The portion of the $\mathrm{CO}_{2}$ and $\mathrm{H}_{2} \mathrm{O}$ mass replaced by mass of $\mathrm{N}_{2}$ is determined such that the overall initial $\gamma$ of the mixture at the pre-ignition point $\left(10^{\circ} \mathrm{CA} \mathrm{bTDC}\right)$ is matched with that of the air-dilute case. However, the $\mathrm{N}_{2}$-dilute case retains similar oxygen mole percentage as the eEGR-dilute case $\left(\chi_{O 2}=15 \%\right)$. The composition in terms of species mass fractions for the three cases is given in Table 2. Figure 8 shows the burn profile for the $\mathrm{N}_{2}$-dilute case in relation to the burn profiles for the air-dilute and eEGR-dilute cases from the Quasi-D model. It can be seen from Figure 8 that the $\mathrm{N}_{2}$-dilute case has a similar burn profile as the air-dilute case, even though the cases have different levels of $\chi_{O 2}$.

Figure 9 shows for the three cases the variation in average $\gamma$ with the progress of combustion. It can be seen from Figure 9 that the $\gamma$ is matched for the air-dilute and $\mathrm{N}_{2}$-dilute cases through the combustion process. It must also be noted that the $\mathrm{N}_{2}$-dilute case has the same initial temperature distribution as the eEGR-dilute case, which is higher than that of the air-dilute case. This indicates that with the overall initial reactivity stratification matched for the air and eEGR-dilute cases, the difference in $\gamma$ between the air- and eEGR-dilute cases shown here is the primary factor in the eEGRdilute case having a $10 \%$ longer combustion duration than the air-dilute case, and that the lower $\gamma$ for the eEGR case acts to reduce the rate of polytropic compression-driven temperature increase in the unburned gas.

\section{Conclusions}

1. External EGR (eEGR) can be used at a given ignition timing in HCCI to increase combustion duration relative to dilution with excess air. The CFD predictions showed a $10 \%$ increase in combustion duration when diluting with eEGR compared to air dilution.

2. Full-cycle CFD simulations that capture breathing and stratification effects arising from trapped residuals indicate that the compositional stratification (for this low-residual engine), in terms of fuel to oxygen ratio and oxygen mole fraction, has a much smaller effect on reactivity 
stratification compared to the thermal stratification of the charge. The thermal stratification of the air- and eEGR-dilute charges, as measured by the $2 \sigma$ of the respective temperature distributions, was within $4 \mathrm{~K}$ for the conditions studied.

3. The pre-ignition reactivity distribution (which is defined in this work in terms of local ignition delays computed within the CFD domain) was nearly identical for the two dilution methods studied. These similar reactivities were obtained near the start of ignition by offsetting the lower oxygen concentration of the eEGR case, which decreases reactivity, with higher overall charge temperatures, which increases reactivity.

4. Considering the nearly identical mixture reactivity with both diluents, the small differences in combustion duration at constant ignition timing are due to the differences in the ratio of specific heats between the two charges resulting in a reduced rate of polytropic compression work transfer from the earlier to later burning regions with eEGR dilution. This was demonstrated by substituting some of the eEGR with $\mathrm{N}_{2}$ in the Quasi-D simulations to match $\gamma$ of the air-dilute case.

\section{Acknowledgments}

The authors would like to acknowledge Dr. Aris Babajimopoulos, Prof. Hong Im, Prof. Jim Driscoll and Prof. Claus Borgnakke for valuable insights. We thank Dr. Laura Olesky for sharing her experimental HCCI data and Rob Middleton for parallelizing the chemistry calculations in the CFD code. This material is based upon work supported by the Department of Energy [National Energy Technology Laboratory] under Award Number(s) DE-EE0003533. This work is performed as a part of the ACCESS project consortium (Robert Bosch LLC, AVL Inc., Emitec Inc., Stanford University, University of Michigan) under the direction of PI Hakan Yilmaz and Co-PI Oliver Miersch-Wiemers, Robert Bosch LLC.

\section{References}

[1] P.M. Najt, D. E. Foster D. E., SAE Technical Paper 830264.

[2] R.H. Thring, SAE Technical Paper 892068.

[3] M. Christensen, B. Johansson, SAE Technical Paper 2000-01-1835. 
[4] J. Olsson, P. Tunestal, J. Ulfvik, B. Johansson, SAE Technical Paper 2003-01-0743.

[5] M. Sjöberg, J.E. Dec, W. Hwang, SAE Technical Paper 2007-01-0207.

[6] J.E. Dec, M. Sjöberg, W. Hwang, SAE Technical Paper 2009-01-2665.

[7] J.E. Dec, SAE Technical Paper 2002-01-1309.

[8] M. Sjöberg, J.E. Dec, Proc. Combust. Inst. 33 (2011) 3031-3038.

[9] J. Kodavasal, Effect of charge preparation strategy on HCCI combustion, PhD thesis, University of Michigan, Ann Arbor, Michigan, USA, 2013.

[10] L.M. Olesky, J. Vavra, D. Assanis, A. Babajimopoulos, J. Eng. Gas Turbines Power 134 (11) (2012) 112801.

[11] L.M. Olesky, J.B. Martz, G.A. Lavoie, J. Vavra, D.N. Assanis, A. Babajimopoulos, Appl. Energy 105 (2013) 407-411.

[12] J. Kodavasal, G. A. Lavoie, J. B. Martz, D. N. Assanis, Submitted to Combustion and Flame (2014) CNF-D-14-00198.

[13] M. Mehl, J.Y. Chen, W.J. Pitz, S.M. Sarathy, C.K. Westbrook, Energy Fuels 25 (11) (2011) 5215-5223.

[14] A. Babajimopoulos, D.N. Assanis, D.L. Flowers, S.M. Aceves, R.P. Hessel, Int. J. Engine Res. 6 (5) (2005) 497-512.

[15] J. Kodavasal, S. Keum, A. Babajimopoulos, Combust. Theor. Model. 15 (6) (2011) 893-910.

[16] J. Kodavasal, C. Kolodziej, S. Ciatti, S. Som, submitted to the ASME Fall IC Engine Conference, Columbus, IN, Oct. 19-22, 2014.

[17] A. Dubreuil, F. Foucher, C. Mounaim-Rousselle, G. Dayma, P. Dagaut, Proc. Combust. Inst. 31 (2) (2007) 2879-2886.

[18] P. Dagaut, A. Nicolle, Combust. Flame 140 (3) (2005) 161-171.

[19] G.A. Lavoie, J. Martz, M. Wooldridge, D. Assanis, Combust. Flame 157 (6) (2010) 1106-1110.

[20] X. He, M.T. Donovan, B.T. Zigler, T.R. Palmer, S.M. Walton, M.S. Wooldridge, A. Atreya, Combust. Flame 142 (3) (2005) 266-275. 
[21] S. S. Goldsborough, Combust. Flame 156 (6) (2009) 1248-1262.

[22] N. Dronniou, J. Dec, SAE Int. J. Engines 5(3) (2012).

[23] J. Kodavasal, M.J. McNenly, A. Babajimopoulos, S.M. Aceves, D.N. Assanis, M.A. Havstad, D.L. Flowers, Int. J. Engine Res. 14 (5) (2013) 416-433. 
Table 1.

Engine Specifications

Cylinder Displacement (L)

0.550

Bore/Stroke (mm)

86.0/94.6

Connecting Rod Length (mm)

152.2

Compression Ratio

$12.50: 1$

IVC/EVO

$130^{\circ} \mathrm{bTDC} / 148^{\circ} \mathrm{aTDC}$

Initial Swirl Ratio

0.3 
Table 2.

Composition by mass fractions at $10^{\circ} \mathrm{CA}$ bTDC imposed to Quasi-D model

\begin{tabular}{llll}
\hline Case & Air-dilute & eEGR-dilute & $\mathrm{N}_{2}$-dilute \\
\hline Fuel & 0.0213 & 0.0215 & 0.0215 \\
$\mathrm{O}_{2}$ & 0.2174 & 0.1653 & 0.1653 \\
$\mathrm{~N}_{2}$ & 0.7484 & 0.7372 & 0.7980 \\
$\mathrm{CO}_{2}$ & 0.0092 & 0.0545 & 0.0109 \\
$\mathrm{H}_{2} \mathrm{O}$ & 0.0036 & 0.0215 & 0.0043 \\
\hline
\end{tabular}




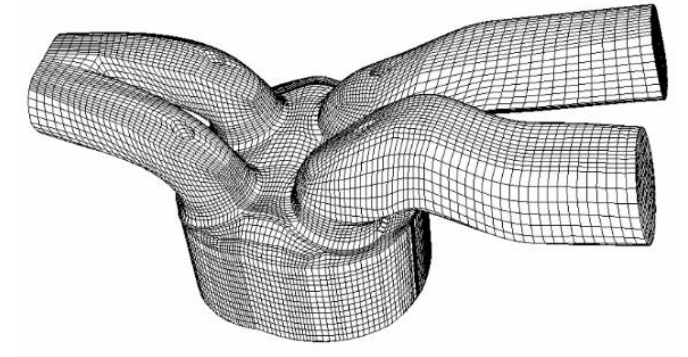

Figure 1. Computational mesh of the engine used in the simulations

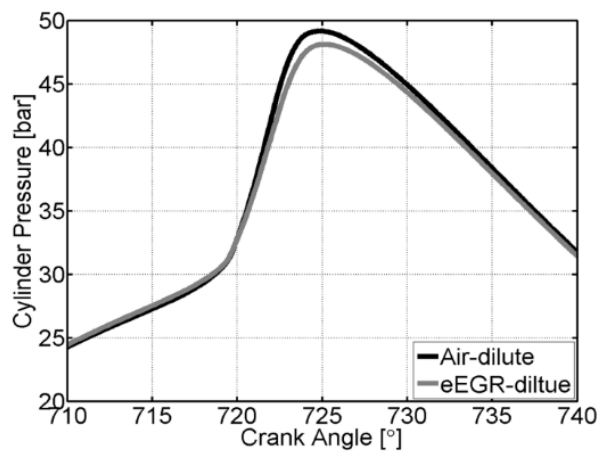

Figure 2. In-cylinder pressure traces from the reacting CFD simulations

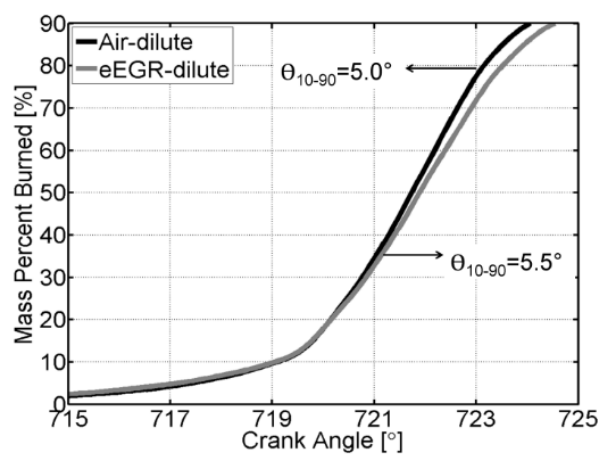

Figure 3. Mass percentage burned profiles from the reacting CFD simulations 

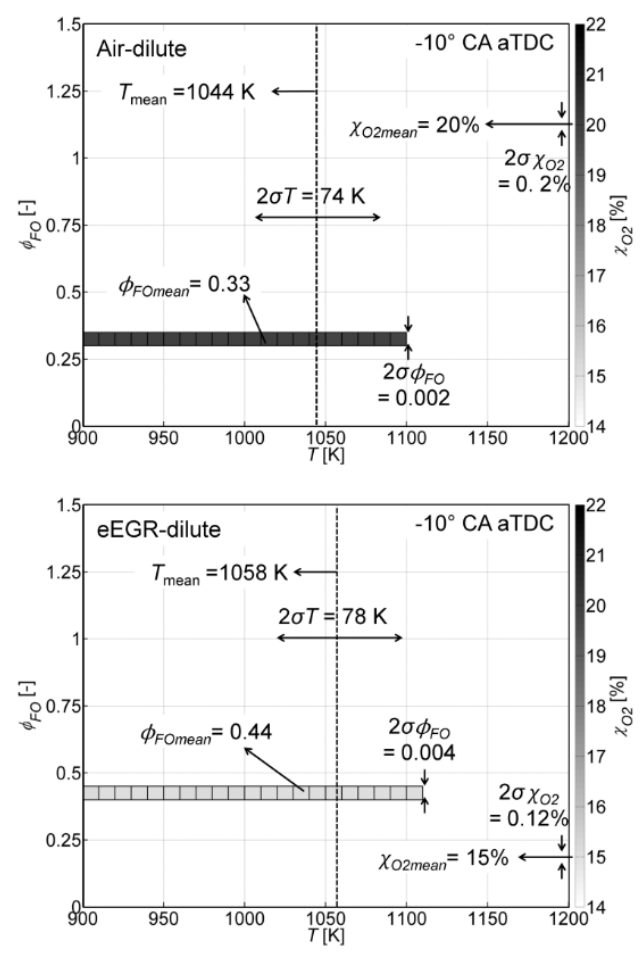

Figure 4. Pre-ignition reaction space $\left(10^{\circ} \mathrm{CA}\right.$ bTDC) for the air-dilute case (top) and the eEGRdilute case (bottom) from CFD simulations

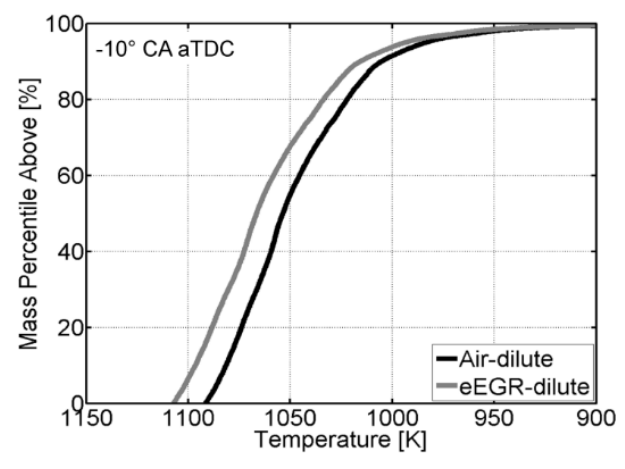

Figure 5. Pre-ignition ( $10^{\circ} \mathrm{CA}$ bTDC) temperature distribution from the CFD simulations for the airdilute and the eEGR-dilute cases (note that temperature axis is in the reverse direction) 


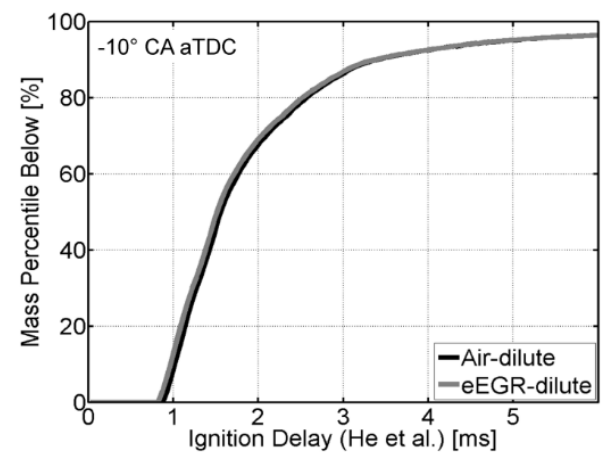

Figure 6. Pre-ignition ( $10^{\circ} \mathrm{CA}$ bTDC) reactivity distribution from the CFD simulations for the airdilute and the eEGR-dilute cases

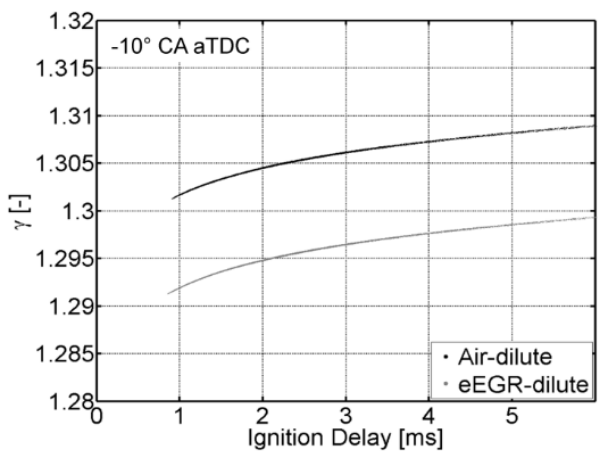

Figure 7. Pre-ignition ( $10^{\circ} \mathrm{CA}$ bTDC) distribution of $\gamma$ from the CFD simulations for the air-dilute and the eEGR-dilute cases

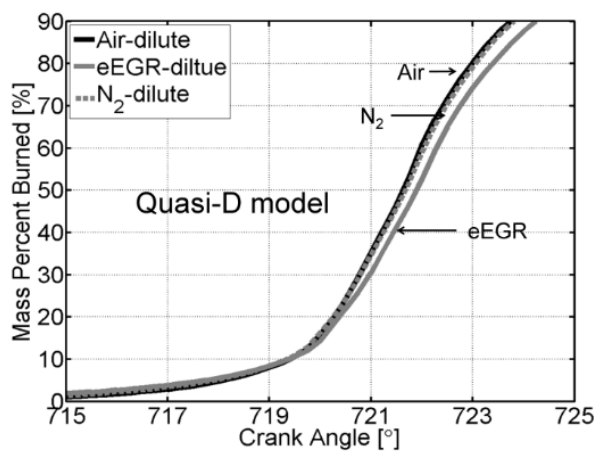

Figure 8. Application of the Quasi-D model to decouple thermodynamic effects; burn profiles corresponding to air dilution, eEGR dilution and $\mathrm{N}_{2}$ dilution shown 


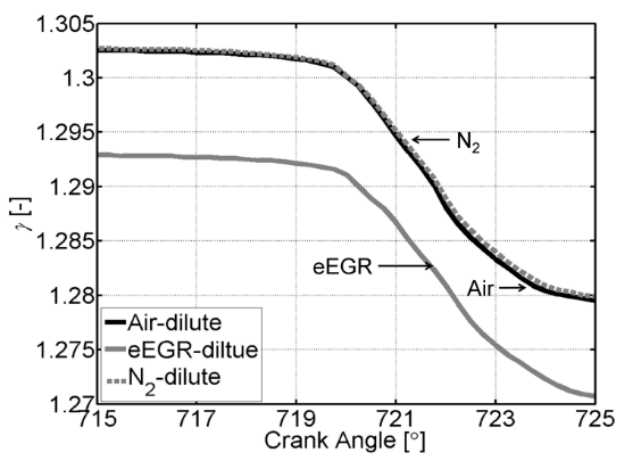

Figure 9. Variation of $\gamma$ with the progress of combustion in the Quasi-D model 


\section{LIST OF FIGURE CAPTIONS}

Figure 1: Computational mesh of the engine used in the simulations

Figure 2: In-cylinder pressure traces from the reacting CFD simulations

Figure 3: Mass percentage burned profiles from the reacting CFD simulations

Figure 4: Pre-ignition reaction space $\left(10^{\circ} \mathrm{CA}\right.$ bTDC) for the air-dilute case (top) and the eEGRdilute case (bottom) from CFD simulations

Figure 5: Pre-ignition $\left(10^{\circ} \mathrm{CA}\right.$ bTDC) temperature distribution from the CFD simulations for the airdilute and the eEGR-dilute cases (note that temperature axis is in the reverse direction)

Figure 6: Pre-ignition $\left(10^{\circ} \mathrm{CA}\right.$ bTDC) reactivity distribution from the CFD simulations for the airdilute and the eEGR-dilute cases

Figure 7: Pre-ignition ( $10^{\circ} \mathrm{CA}$ bTDC) distribution of $\gamma$ from the CFD simulations for the air-dilute and the eEGR-dilute cases

Figure 8: Application of the Quasi-D model to decouple thermodynamic effects; burn profiles corresponding to air dilution, eEGR dilution and $\mathrm{N}_{2}$ dilution shown

Figure 9: Variation of $\gamma$ with the progress of combustion in the Quasi-D model 